\title{
PCCP
}

ARTICLE TYPE

Cite this: DOI: $00.0000 / x x x x x x x x x x$

\section{Excitation Energy Transport in DNA modelled by Multi- Chromophoric Field-Induced Surface Hopping}

\author{
Matthias Wohlgemuth, ${ }^{a}$ and Roland Mitrić ${ }^{* a}$
}

Received Date

Accepted Date

DOI: 00.0000/xxxxxxxxxx

\begin{abstract}
Absorption of ultraviolet light is known as a major source of carcinogenic mutations of DNA. The underlying processes of excitation energy dissipation are yet not fully understood. In this work we provide a new and generally applicable route for studying the excitation energy transport in multichromophoric complexes at an atomistic level. The surface-hopping approach in the frame of the extended Frenkel exciton model combined with $\mathrm{QM} / \mathrm{MM}$ techniques allowed us to simulate the photodynamics of the alternating $(\mathrm{dAdT})_{10}$ : (dAdT) $)_{10}$ double-stranded DNA. In accordance with recent experiments, we find that the excited state decay is multiexponential, involving a long and a short component which are due to two distinct mechanisms: formation of long-lived delocalized excitonic and charge transfer states vs. ultrafast decaying localized states resembling those of the bare nucleobases. Our simulations explain all stages of the ultrafast photodynamics including initial photoexcitation, dynamical evolution out of the Franck-Condon region, excimer formation and nonradiative relaxation to the ground state.
\end{abstract}

\section{Introduction}

The fact that UV radiation can trigger carcinogenic mutations in DNA due to the absorption of light has been well established for a long time $\mathrm{e}^{1+3}$. Understanding DNA excited-state dynamics is thus essential for determining how photolesions are formed. Therefore, the excited states and their relaxation in the monomeric nucleobases as well as in DNA oligo- and polymers have been subject to numerous experimental and theoretical studies in the past decades ${ }^{4}$. While the excited state lifetime of the monomeric nucleobases is remarkably short $(<1 \mathrm{ps})$, the deactivation in nucleobase multimers, despite the additional degrees of freedom, takes place on the timescale from 10 to several $100 \mathrm{ps} 8$ 12. Excitonic 5 (8) 10/13,18 and delocalized charge transfer (CT) $5 / 6 \mid 10] 12[19$ states have been made responsible for this difference and the role of these states has been discussed extensively on the basis of experimental and theoretical $14|20| 29$ work. The processes taking place in the UV excited DNA are until now not fully understood and this topic is at the very frontier of the current research 15 .

Crespo-Hernández et al. found that the transient absorption signals, which they associated with intra-strand excimers, decay with time constants of 150 ps for the homopolymeric AdenineThymine (AT) double-stranded DNA (dsDNA) and 51 ps for the

\footnotetext{
${ }^{a}$ Institut für Physikalische und Theoretische Chemie, Julius-Maximilians-Universität Würzburg, Emil-Fischer-Str. 42, 97074 Würzburg, Germany. E-Mail: roland.mitric@uni-wuerzburg.de

${ }^{b}$ Address, Address, Town, Country.

$\dagger$ Electronic Supplementary Information (ESI) available: [details of any supplementary information available should be included here]. See DOI: 00.0000/00000000.
}

alternating AT dsDNA 12 . Transient absorption spectra for similar duplexes were reported and the fingerprint of exciton states was detected 30 . Fluorescence anisotropy data obtained for various dsDNA revealed that ultrafast $(<100 \mathrm{fs}$ ) energy transfer is made possible in these systems after the population of FranckCondon exciton states $8|31| 32$. Fluorescence from excited states emitting at longer wavelengths than those of the monomeric chromophores has been found for homopolymeric Guanine-Cytosine (GC) double-stranded $\mathrm{DNA}^{32}$ and alternating AT duplexes $9[16$. Many of those experiments suggest that fast monomeric deactivation channels as well as long-living exciton states coexist and the observed multi-exponential decays are results of this 16 .

From the theoretical point of view, highly correlated ab-initio methods have been employed to simulate absorption spectra in the Franck-Condon (FC) region e.g. by Nogueira et al. for alternating AT tetramers 26 and Spata et al. ${ }^{22}$ for stacked nucleobase trimers of different composition. They used a QM/ MM approach where the quantum region was treated with the ab initio ADC(2) method for the excited states and included up to four stacked bases in the quantum region. They concluded that delocalization does not occur over more than two bases, while CT states are located at higher energies than the bright states in the FC region. Coupling between locally excited and CT states was also predicted 2324. Dinucleotides in the gas phase and in solution were also studied by Plasser et al., and it was found that excitonic states exist with remarkably short intermolecular separation and with CT character 25 . Calculations on adenine tetramers including backbone and solvent suggested that the excited state dynamics 
of stacked homopolymeric bases is modulated by the interplay of bright monomeric excited states, $\pi \pi^{*}$ excitonic, and CT states 33 .

A recent study of the potential energy surface at QM(CASPT2//CASSCF)/MM level, having a stacked thymine dimer in the QM region, suggests that an ultrafast intrabase $\pi \pi^{*} \rightarrow S_{0}$ transition reached by three different pathways is responsible for the short component observed in time-resolved experiments 20 . A fourth decay channel was found as well, which is however blocked by a $0.5 \mathrm{eV}$ barrier arising due to a stabilizing inter-base excitonic state. Similarly, Szabia et al. recently reported in a QM/MM study a UV-induced repair mechanism for Cyclobutane pyrimidine dimers (CpDs) formed by thymine dimerization 34 . There are also several QM/MM studies combined with ground-state molecular dynamics (MD) simulations using accurate ab-initio and semiempirical methods 21,24,35.

However, these MD studies focus on the geometries close to the FC region, and thus on the photochemistry in the early stages of the excitation only. An explicit treatment of nonadiabatic dynamics was addressed by Martinez and co-workers by combining an exciton model with Tully's fewest switching algorithm ${ }^{36}$ in the frame of time-dependent density functional theory to study the excitation energy transport in the light-harvesting complex $\mathrm{II} 37 / 38$. In this model the chromophores are coupled by coulombic dipole-dipole interaction and the electronic wave function is represented in the basis of excitonic eigenstates obtained from the product basis of ground- and singly-excited adiabatic wave functions of the individual chromophores. Recently, this approach was extended by Gonzalez and co-workers by introducing an QM/MM-like electrostatic embedding scheme ${ }^{39}$, which allows for the simulation of chemically bonded chromophores as well. Both methods, however, are lacking the explicit inclusion of the irradiating laser pulse and the excited state dynamics are usually initiated by setting one of the monomers into the excited state close to the absorption band.

In the present work, we combine QM/MM techniques with a state-of-the-art field-induced surface-hopping approach $40 / 41$ in the frame of an extended exciton model, allowing us to investigate the excitation energy transport and non-radiative relaxation including all degrees of freedom. As a model we have chosen the double-stranded B-DNA of 20 alternating Desoxyadenosines (dA) and Desoxythymidines (dT) per strand, where the central 6 base pairs are included in the QM region. This is, to the best of our knowledge, the largest DNA model considered in a QM/MM MD study so far and further the first application to the real-time photodynamics of dsDNA. The chosen model allows us to investigate both intra-strand processes due to $\pi$-stacking as well as inter-strand ones like coupled proton/electron transfer. With our simulations we wish to shed more light on the photochemical processes taking place in the first picoseconds after excitation of DNA and present a versatile general tool for simulating excitation energy transport processes in multi-chromophoric complexes.

The presented method is generally and widely applicable and can serve e.g. for the investigation of excitation energy transfer processes occurring in light-harvesting complexes 42 , biological imaging applications in fluorescent proteins $45 \mid 47$ or the study and design of photoactivatable drugs as used in photodynamic therapy (PDT) 48 . 51

\section{Multi-chromophoric Field-induced Sur- face Hopping (McFISH)}

In the following we describe the theoretical formulation of our multi-chromophoric field-induced surface hopping (McFISH) method, which is employed here to simulate the excitation energy transport (EET) and non-radiative relaxation in a DNA doublestrand. In the present work, we follow a hybrid multi-quantum classical QM/MM approach, where multiple subsets of nucleobases (e.g. a stacked base pair) are described fully quantum mechanically (QM), and the backbone, solvating water molecules, ions as well as the other nucleobases are described by a classical force field (MM). In this way, all electronic states within the stacked pair containing four bases are treated fully quantummechanically, which automatically provides the correct description of both local excited as well as charge transfer states. Each of these QM subsystem is individually electrostatically embedded into the environment composed of all other QM subsystems and the MM system by including the atomic point charges into the one-particle hamiltonian. The exact partitioning of the system is presented in Fig. 1 .

In following, we summarize the theoretical approach using the following notation: $\mathbf{R}$ denote spatial positions of the nuclei and r spatial electron coordinates. Capital indices $I$ and $J$ denote the subsystems, such that $\mathbf{R}_{I}$ denotes the subset of nuclear coordinates of in subsystem $I$. Small indices $a, b \ldots$ denote excitations within a single chromophoric subunit.

QM/MM exciton model. As in the conventional hybrid quantum/molecular mechanics (QM/MM) approach 52 the total energy of the system is obtained as:

$$
E=\left\langle\Psi_{M C}\left|\mathbf{H}_{M C}\right| \Psi_{M C}\right\rangle+E_{M M}\left(\mathbf{R}_{M M}\right)+E_{Q M / M M}\left(\mathbf{R}_{1}, \ldots, \mathbf{R}_{N}, \mathbf{R}_{M M}\right)
$$

where the first term is the QM energy of the multi-chromophoric complex (MC), the second term is the classical force field energy of the MM region and the third term is the interaction between the chromophoric complex and the MM system, respectively. In the electrostatic embedding scheme ${ }^{53}$ used here the Coulombic interaction of the QM and MM system is included in $\mathbf{H}_{M C}$ as a one-electron operator and $E_{Q M / M M}$ reduces to the Van-der-Waals interaction and the classical bonding interaction at the boundary of QM and MM system only.

We consider the MC to be built from $N$ building blocks, which we will further refer as subunits, subsystems or monomers. In the exciton model the electronic Hamiltonian $\mathbf{H}_{M C}$ of such a chromophoric complex irradiated by an external laser field can be composed from the monomeric Hamiltonian of each subsystem $\mathbf{H}_{I}\left(\equiv \mathbf{H}\left(\mathbf{r}_{I}, \mathbf{R}_{I}\right)\right)$, the pairwise interaction $\mathbf{V}_{I J}{ }^{55}$ and the interaction with the laser field $\mathbf{V}^{\text {ext }}$ :

$$
\mathbf{H}_{M C}=\sum_{I} \mathbf{H}_{I} \otimes \mathbf{1}_{I}+\frac{1}{2} \sum_{I} \sum_{J \neq I} \mathbf{V}_{I J} \otimes \mathbf{1}_{I J}+\sum_{I} \mathbf{V}_{I}^{e x t}(t) \otimes \mathbf{1}_{I},
$$

where $\mathbf{1}_{I}$ and $\mathbf{1}_{I J}$ are identity operators acting on the electrons of all monomers, except the $I$-th or the $I$-th and $J$-th, respectively.

In Eq. (2) the first two terms are time-independent and 

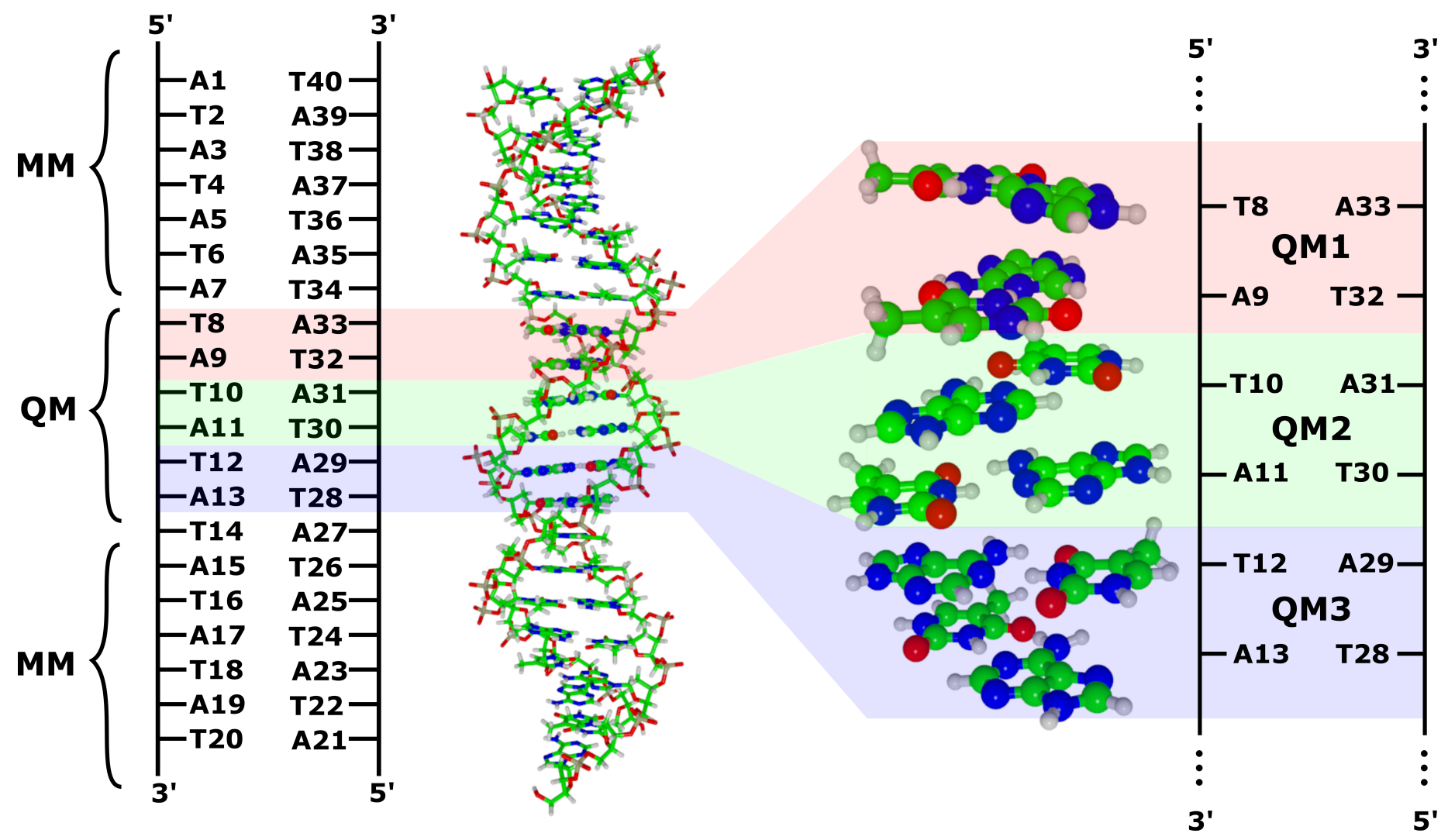

Fig. 1 Partitioning of the $(\mathrm{dAdT})_{10}:(\mathrm{dAdT})_{10}$ double-strand DNA-helix (environment of water and ions not shown). Left: Schematic and 3-dimensional structure of the whole system containing the 20 alternating dA : dT base pairs. The system is first divided into a QM part, containing 6 A : T base pairs, which is treated at the semiempirical OM2/MR-Cl level and an MM part, consisting of the residual backbone, nucleobases, water and ions, respectively. Right: The 6 base pairs treated quantum mechanically. The red, green and blue areas indicate the partitioning into the 3 subsystems, where each is computed separately and is embedded into the charge field of all surrounding structures.

are completely determined by the structure of the individual monomers. Therefore the natural basis for solving the timedependent Schrödinger equation (TDSE) with the Hamiltonian (Eq. (2)) is the basis spanned by the eigenstates of the timeindependent part of Eq. (2).

The $\mathbf{V}_{I J}$ operator in Eq. (2) represents the interaction between the chromophores at the sites $I$ and $J$, respectively:

$$
\mathbf{V}_{I J}=\mathbf{V}_{I J}^{(n n)}+\mathbf{V}_{I J}^{(e n)}+\mathbf{V}_{I J}^{(e e)}
$$

including the nuclear-nuclear $\mathbf{V}_{I J}^{(n n)}\left(\equiv \mathbf{V}\left(\mathbf{R}_{I}, \mathbf{R}_{J}\right)\right.$ ), the electronnuclear $\mathbf{V}_{I J}^{(e n)}\left(\equiv \mathbf{V}\left(\mathbf{r}_{I}, \mathbf{R}_{J}\right)+\mathbf{V}\left(\mathbf{r}_{J}, \mathbf{R}_{I}\right)\right)$ and the electron-electron $\mathbf{V}_{I J}^{(e e)}\left(\equiv \mathbf{V}\left(\mathbf{r}_{I}, \mathbf{r}_{J}\right)\right)$ contributions. Since $\mathbf{V}_{I J}^{(e e)}$ depends on the electron coordinates of two subsystems, the total wave function of the MC cannot be obtained easily in a self-consistent way.

In order to make such an exciton model practical for calculations in large systems, we introduce the following approximations: (i) the wave functions of the individual monomers are approximated by the wave functions of the isolated monomers embedded in the field of charges of the other monomers and solvent, (ii) no exchange of electrons is allowed between the subunits but does occur between individual chromophores within the subunit, and (iii) the molecular orbitals from different monomers are con- sidered to be orthogonal, which is strictly not exact, since the orbitals are determined independently. Thus we expand the total multi-chromophoric wave function $\left|\Psi_{M C}\right\rangle$ into the direct products basis of the electronic wave functions of the individual QM subsystems:

$$
\left|\phi_{a b \ldots z}\right\rangle=\left|\varphi_{a}^{1}\right\rangle \otimes\left|\varphi_{b}^{2}\right\rangle \otimes \ldots \otimes\left|\varphi_{z}^{N}\right\rangle=\left|\prod_{I} \varphi_{k_{I}}^{I}\right\rangle
$$

where superscripts $1,2, \ldots, N$ denote the monomer index number within the MC, the indices $a, b, \ldots z$ are running over all included electronic states for each monomer (e.g. $a \in\left[0, \mathscr{N}_{1}-1\right]$, $\left.b \in\left[0, \mathscr{N}_{2}-1\right], \ldots\right)$ and $k_{I}$ is the index of the electronic state of the $I$-th monomer in the set $a b \ldots z$. Since a QM subsystems itself may contain several chromophores (e.g. four in the case of a stacked base pair) such a direct product basis automatically includes both delocalized Frenkel exciton states as well as charge transfer states.

Each eigenstate $\left|\varphi_{i}^{I}\right\rangle$ in Eq. (4) fulfills the general orthogonality relation $\left\langle\varphi_{i}^{I} \mid \varphi_{j}^{J}\right\rangle=\delta_{i j} \delta_{I J}$ and satisfies the time-independent Schrödinger equation $\mathbf{H}_{I}\left|\varphi_{i}^{I}\right\rangle=\mathscr{E}_{i}^{I}\left|\varphi_{i}^{I}\right\rangle$, with $\mathscr{E}_{i}^{I}$ being the $i$-th electronic state energy of the $I$-th monomer. In the electrostatic embedding scheme the monomeric Hamiltonian of the $I$-th subsys- 
tem in the manifold of $\mathscr{N}_{I}$ electronic states can be written as:

$$
\mathbf{H}_{I}=\sum_{i}^{\mathscr{N}_{I}}\left[\mathscr{H}_{i}^{I}+V_{i}^{I}\left(\mathbf{R}_{M M}\right)+\sum_{J \neq I} V_{i}^{I}\left(\mathbf{R}_{J}\right)\right]\left|\varphi_{i}^{I}\right\rangle\left\langle\varphi_{i}^{I}\right|,
$$

where $\mathscr{H}_{i}^{I}$ is the unembedded Hamiltonian matrix element of the $i$-th electronic state. The terms $V_{i}^{I}\left(\mathbf{R}_{M M}\right)$ and $V_{i}^{I}\left(\mathbf{R}_{J}\right)$ represent the one-electron and nuclear contributions to the wave function of the $I$-th subsystem due to interaction with the other subsystems (indicated as $\mathbf{R}_{J}$ ) and the MM region $\left(\mathbf{R}_{M M}\right)$, respectively:

$$
V_{i}^{I}\left(\mathbf{R}_{K}\right)=\left\langle\varphi_{i}^{I}\left|\sum_{v \in I} \sum_{\alpha \in K} \frac{Q_{\alpha}}{\left|\mathbf{r}_{v}^{I}-\mathbf{R}_{\alpha}^{K}\right|}\right| \varphi_{i}^{I}\right\rangle+\sum_{\substack{\alpha \in I \\ \beta \in K}} \frac{Q_{\alpha} Q_{\beta}}{\left|\mathbf{R}_{\alpha}^{I}-\mathbf{R}_{\beta}^{K}\right|}
$$

Here $K$ stands for either one of the other monomers or the MM system, the index $v$ runs over electron positions, and the indices $\alpha$ and $\beta$ over nuclear coordinates of the respective subsystems.

Since the nuclear contributions $\mathbf{V}_{I J}^{(n e)}$ and $\mathbf{V}_{I J}^{(n n)}$ in Eq. (3) are shifted into the one-electron operator of the monomeric Hamiltonian (Eq. (5)), Eq. (3) is reduced to classical Van-der-Waals interactions and pure electronic contributions $\mathbf{V}_{I J}^{(e e)}$. Using the orthogonality of the monomeric electronic states the matrix elements of the $\mathbf{V}_{I J}^{(e e)}$ operator reduce to pure electronic contributions between 4 electronic states:

$$
\mathbf{V}_{I J}^{(e e)}=\sum_{\substack{k, l \\ k^{\prime} \neq k \\ l^{\prime} \neq l}} J_{k l, k^{\prime} l^{\prime}}^{I J}\left|\varphi_{k}^{I} \varphi_{l}^{J}\right\rangle\left\langle\varphi_{k^{\prime}}^{I} \varphi_{l^{\prime}}^{J}\right|
$$

where $J_{k l, k^{\prime} l^{\prime}}^{I J}$ are the Coulomb integrals. These can be calculated from the transition densities $\rho_{k k^{\prime}}^{I}$ and $\rho_{l l^{\prime}}^{J}$ according to:

$$
\begin{aligned}
J_{k l, k^{\prime} l^{\prime}}^{I J} & =\left\langle\varphi_{k}^{I}\left|\otimes\left\langle\varphi_{l}^{J}\left|\sum_{v, \lambda} \frac{1}{\left|\mathbf{r}_{v}^{I}-\mathbf{r}_{\lambda}^{J}\right|}\right| \varphi_{k^{\prime}}^{I}\right\rangle \otimes\right| \varphi_{l^{\prime}}^{J}\right\rangle \\
& =\int \mathrm{d} \mathbf{r}_{1}^{I} \int \mathrm{d} \mathbf{r}_{1}^{J} \frac{\rho_{k k^{\prime}}^{I}\left(\mathbf{r}_{1}^{I}\right) \rho_{l l^{\prime}}^{J}\left(\mathbf{r}_{1}^{J}\right)}{\left|\mathbf{r}_{1}^{I}-\mathbf{r}_{1}^{J}\right|} .
\end{aligned}
$$

where the indices $\lambda$ and $v$ run over electron positions of the monomer $I$ and $J$, respectively, and the transition densities are obtained within the basis of the individual monomers by integrating all $\left(N_{I}\right)$ electron coordinates except the first $\mathbf{r}_{1}^{I}$ :

$$
\rho_{k k^{\prime}}^{I}\left(\mathbf{r}_{1}^{I}\right)=N_{I} \int \mathrm{d} \mathbf{r}_{2}^{I} \cdots \int \mathrm{d} \mathbf{r}_{N_{I}}^{I} \varphi_{k}^{I *}\left(\mathbf{r}^{I}\right) \varphi_{k^{\prime}}^{I}\left(\mathbf{r}^{I}\right)
$$

Since the evaluation of the two-center integral $J_{k l, k^{\prime} l^{\prime}}^{I J}$ is challenging for extended systems, there are several approximations available: (i) replacing the transition density $\rho$ by atomic transition charges obtained from fitting the transition density to the electrostatic potential 56 , (ii) the transition density cube (TDC) method, where $\rho$ is represented on a 3-dimensional grid and the overlap is calculated numerically on that grid 57 and (iii) using the transition dipole approximation (TDA) ${ }^{58}$. In this work we employ the TDA, which is the most efficient approximation and can be used if the molecules interacting with each other are sufficiently well separated and short-range contributions can be neglected. However note that any of the available approximations can be used within the presented methodology.
Within the TDA Eq. (8) is further represented by a multipole expansion up to the second order and the integral for an uncharged molecule is reduced to

$$
J_{k l, k^{\prime} l^{\prime}}^{I J} \approx \frac{\mu_{k k^{\prime}}^{I} \cdot \mu_{l l^{\prime}}^{J}}{\left|\mathbf{R}_{I J}\right|^{3}}-3 \frac{\left(\mu_{k k^{\prime}}^{I} \cdot \mathbf{R}_{I J}\right)\left(\mu_{l l^{\prime}}^{J} \cdot \mathbf{R}_{I J}\right)}{\left|\mathbf{R}_{I J}\right|^{5}},
$$

where $\mathbf{R}_{I J}$ is the vector connecting the center of coordinates of the two monomers and $\mu_{k k^{\prime}}^{I}$ is the transition dipole moment for the excitation from electronic state $k$ to $k^{\prime}$ within the monomer $I$ :

$$
\mu_{k k^{\prime}}^{I}=\left\langle\varphi_{k}^{I}\left|\sum_{v \in I} \mathbf{r}_{v}^{I}\right| \varphi_{k^{\prime}}^{I}\right\rangle
$$

We are aware of the fact that the TDA probably overestimates the excitonic coupling of the chromophores 59 . However, we have chosen this approximation for practical reasons in the simulation of the field-driven dynamics. Fitting the transition charges to the electrostatic potential in the manifold of all excited states is computationally expensive and computing the transition density on the grid needs careful calibration of the chosen grid, especially in the context of the molecular dynamics simulations as performed in this work. In contrast, the computation of transition dipole moments is relatively cheap and is already required to compute the coupling to the external laser field in the McFISH method, as described later in this section. In order to compensate the caveats to this methodological choice, we have chosen a model having two base pairs included in each monomeric unit, such that short-range contributions are partially accounted for.

Exciton state gradients and nuclear dynamics. In order to propagate the nuclear trajectories in the excitonic states as defined by Eq. (4) we solve Newton's equations of motion:

$$
\mathbf{F}=-\nabla_{\mathbf{R}} E_{a b \ldots z}
$$

where $E_{a b \ldots z}$ is the total energy of the excitonic basis state $\left|\phi_{a b \ldots z}\right\rangle$ defined as:

$$
\begin{aligned}
E_{a b \ldots z} & =\sum_{I}\left\langle\varphi_{i}^{I}\left|\mathbf{H}_{I}\right| \varphi_{i}^{I}\right\rangle+E_{M M}\left(\mathbf{R}_{M M}\right)+E_{Q M / M M}\left(\mathbf{R}_{1}, \ldots, \mathbf{R}_{N}, \mathbf{R}_{M M}\right) \\
& =\sum_{I} \mathscr{E}_{i}^{I}\left(\mathbf{R}_{I}\right)+E_{M M}\left(\mathbf{R}_{M M}\right)+E_{Q M / M M}\left(\mathbf{R}_{1}, \ldots, \mathbf{R}_{N}, \mathbf{R}_{M M}\right)
\end{aligned}
$$

where $\mathscr{E}_{i} I$ is the energy of the $I$-th monomer being in the $i$-th electronic state, which is obtained from quantum chemical calculations. By decomposing the total nuclear gradient $\nabla_{\mathbf{R}}$ with respect to all nuclear coordinates of the whole system $\mathbf{R}$ into components of the individual subunits (QM monomers $\left(\mathbf{R}_{1}, \ldots, \mathbf{R}_{N}\right)$ as well as the MM part $\mathbf{R}_{M M}$ ), the gradient of Eq. (13) can be written as:

$$
\nabla_{\mathbf{R}} E_{a b \ldots z}=\left(\begin{array}{c}
\nabla_{R_{1}} \mathscr{E}_{a} \\
\nabla_{R_{2}} \mathscr{E}_{b}^{2} \\
\vdots \\
\nabla_{R_{N}} \mathscr{E}_{z} \\
\nabla_{R_{M M}} E_{M M}
\end{array}\right)+\nabla_{\mathbf{R}} E_{Q M / M M}
$$

where the first term can be easily obtained from quantum chemical calculations and $\nabla_{R} E_{Q M / M M}$ are the pair-wise gradients ob- 
tained from van-der-Waals potentials.

Note that in the frame of this formulation the gradients $\nabla J^{I J}$ are neglected, since these terms are small due to the slow variation of the inter-system distance $\mathbf{R}_{I J}$ and the transition dipole moments $\mu_{I}^{k k^{\prime} 60 \mid 61 \text {. }}$.

This is in contrast to the previously published approaches of Martinez $\frac{37 / 38}{38}$ and Gonzalez $\frac{39}{}$, which are based on diagonalization of the Hamiltonian $\mathbf{H}_{M C}$ and nuclear as well as electronic propagation on the resultant adiabatic electronic surfaces. While these two approaches are fully equivalent in a purely quantum mechanical treatment, their semiclassical limits differ. In particular, the nuclear gradients of the adiabatic electronic surfaces include contributions from the gradient of the excitonic coupling, which are absent in our method. However, in the course of the dynamics these coupling gradients are usually small since they depend on the only slowly varying interchromophoric distances and (transition) dipole moments. In addition, the inclusion of these effects is associated with the cost of calculating a large number of forces that increases exponentially with the number of monomers and excited states. More precisely, in the extreme case, gradients of potential energy and excitonic coupling in all electronic states of all monomers are needed. For example, already for a system consisting of 2 monomers with 2 electronic states $g$ and $e$ each, an excitonic wave function like $|e g\rangle+|g e\rangle$ requires 4 quantum chemical gradient calculations in total. Instead, as a great advantage for the construction of the total gradient (14) only a single gradient calculation per monomer is needed and therefore scales linear with the number of monomers. Additionally, the decoupling of the quantum chemical calculations allows for the independent treatment of the individual subsystems and is therefor highly parallelizable. In the current implementation of McFISH all quantum chemical calculations can be performed simultaneously and the results are obtained at once. Note that the off-diagonal terms of $\mathbf{H}_{M C}$ are still included in the propagation of the electronic wave function during the surface hopping procedure, allowing the transport of excitation energy between the individual chromophores of the MC.

We also wish to emphasize that the method presented here includes the description of non-adiabatic effects as well as the early stages of the excitation due to the coupling to the external laser field as shown in the next section.

Excitation energy transport. For the simulation of the excitation energy transport (EET) between the chromophoric subunits we employ a surface-hopping approach ${ }^{36 \mid 62}$ by solving the timedependent Schrödinger equation (TDSE) for the Hamiltonian as defined in Eq. (2). For this purpose, we expand the total (timedependent) wave function in the excitonic basis as defined in Eq. (4):

$$
\left|\Psi_{M C}(t)\right\rangle=\sum_{a b \ldots z} c_{a b \ldots z}(t)\left|\phi_{a b \ldots z}(t ; \mathbf{R}(t))\right\rangle,
$$

with $c_{a b \ldots z}(t)$ being the time-dependent expansion coefficients and the excitonic states being parametrically dependent on the nuclear trajectory $\mathbf{R}(t)$. Inserting Eqs. (15) and (2) into the TDSE yields a set of differential equations for the expansion coefficients

$$
\begin{aligned}
\mathbf{C}=\left[c_{a b \ldots z}, c_{a^{\prime} b \ldots z}, \ldots, c_{a^{\prime} b^{\prime} \ldots z^{\prime}}\right]: & \\
i \dot{\mathbf{C}}(t) & =\mathbf{H}_{M C} \mathbf{C}(t)-i \mathbf{D C}(t) \\
& =\left[\sum_{I}\left(\mathbf{H}_{I}+\mathbf{V}_{I}^{e x t}(t)\right) \otimes \mathbf{1}_{I}+\frac{1}{2} \sum_{J \neq I} \mathbf{V}_{I J} \otimes \mathbf{1}_{I J}-i \mathbf{D}\right] \mathbf{C}(t),
\end{aligned}
$$

where $\mathbf{D}$ is the non-adiabatic coupling matrix, whose elements have the general form:

$$
(\mathbf{D})_{a b \ldots z, a^{\prime} b^{\prime} \ldots z^{\prime}}=\left\langle\phi_{a b \ldots z} \mid \frac{\mathrm{d}}{\mathrm{d} t} \phi_{a^{\prime} b^{\prime} \ldots z^{\prime}}\right\rangle .
$$

In the case that the vibrational modes of the individual monomers are uncoupled the non-adiabatic coupling matrix reduces to:

$$
\mathbf{D}=\sum_{I} \mathbf{D}_{I} \otimes \mathbf{1}_{I}=\sum_{I} \mathbf{1}_{I} \otimes \sum_{k k^{\prime}}^{\mathscr{N}_{I}} \mathbf{d}_{k k^{\prime}}^{I}\left(\mathbf{R}_{I}(t)\right) \cdot \dot{\mathbf{R}}_{I}(t)\left|\varphi_{k}^{I}\right\rangle\left\langle\varphi_{k^{\prime}}^{I}\right|
$$

where $\mathbf{d}_{k k^{\prime}}^{I}$ are the non-adiabatic coupling vectors and $\dot{\mathbf{R}}_{I}$ are the velocities of the nuclei of the $I$-th monomer. The coupling of the individual monomers to the external laser field is described by the time-dependent $\mathbf{V}^{\text {ext }}$ term in Eq. (16). Since we are dealing with a system whose individual components are much smaller than the wavelength of the field, the interaction with the electric field $\mathbf{E}(t)$ can be approximated by a dipole term:

$$
\mathbf{V}_{I}^{e x t}=-\sum_{k k^{\prime}} \mu_{k k^{\prime}}^{I}\left(\mathbf{R}_{I}(t)\right) \cdot \mathbf{E}(t)\left|\varphi_{k}^{I}\right\rangle\left\langle\varphi_{k^{\prime}}^{I}\right|,
$$

where $\mu_{k k^{\prime}}^{I}$ is the transition dipole moment as defined in Eq. (11). Within this study we have used a uniformly polarized Gaussianshaped laser pulse, such that:

$$
\mathbf{E}(t)=\frac{\varepsilon_{0}}{\sqrt{3}}\left(\hat{\mathbf{e}}_{x}+\hat{\mathbf{e}}_{x}+\hat{\mathbf{e}}_{z}\right) \exp \left(-\frac{\left(t-t_{0}\right)^{2}}{2 \sigma^{2}}\right) \sin \left(\omega_{0} t\right),
$$

where $\varepsilon_{0}$ is the amplitude, $t_{0}$ is the center of the pulse, $\sigma$ is the temporal width and $\omega_{0}$ is the central frequency of the pulse. The used parameters can be found in the Computational section. Note that any form of the laser pulse or polarization can be used within this approach ${ }^{63}$ and extensions beyond the electric-field coupling are available 64

The time-dependent Schrödinger equation (16) is integrated in each nuclear time step $\Delta t$ and the electronic state in which the trajectory resides is determined by a stochastic surface hopping algorithm, using hopping probabilities calculated from the change of the quantum electronic state populations $\rho=\mathbf{C C}^{\dagger}$ according to 65 :

$$
P_{\left|\phi_{i}\right\rangle \rightarrow\left|\phi_{f}\right\rangle}=\Theta\left(-\dot{\rho}_{i, i}\right) \Theta\left(\dot{\rho}_{f, f}\right) \frac{-\dot{\rho}_{i, i}}{\rho_{i, i}} \frac{\rho_{f, f} \Delta t}{\sum_{k} \Theta\left(\dot{\rho}_{k, k}\right) \dot{\rho}_{k, k}},
$$

where the $\Theta$ functions are defined to be one for positive arguments and zero otherwise. The indices $i$ and $f$ abbreviate the excitonic states (e.g. $\left|\phi_{a b \ldots z}\right\rangle$ and $\left|\phi_{a^{\prime} b^{\prime} \ldots z^{\prime}}\right\rangle$ ) and the sum runs over all possible excitonic states. If the hopping probability for changing from the excitonic state $\left|\phi_{i}\right\rangle$ to $\left|\phi_{f}\right\rangle$ is greater than a uniformly generated random number a switch to the excitonic state $\left|\phi_{f}\right\rangle$ is made. However, there are several possibilities for state switches: (i) changing from the ground state to an excited 
state (e.g. $\left.\left|\phi_{00 \ldots 0}\right\rangle \rightarrow\left|\phi_{a b \ldots z}\right\rangle\right)$ ), most likely due to excitation by the electric field, (ii) changing from an excited state to the ground state (e.g. $\left.\left|\phi_{a b \ldots z}\right\rangle \rightarrow\left|\phi_{00 \ldots 0}\right\rangle\right)$, due to non-adiabatic relaxation processes, or (iii) a switch of the excitation site (e.g. $\left|\phi_{a^{\prime} b \ldots z}\right\rangle \rightarrow$ $\left.\left|\phi_{a b^{\prime} \ldots z}\right\rangle\right)$. While the first two cases are diabatic events within a single monomer (diabatic hop), the latter one involves state switches in multiple monomers (excitonic hop). Depending on the type of state switch, different energy rescaling mechanism have to be employed.

We assume that all energy originating from the laser pulse is absorbed by the molecule and in the case (i) no velocity rescaling is performed in the occasion of a state switch while the pulse is active. After the pulse has ceased only diabatic hops due to nonadiabatic relaxation processes or excitonic hops due to dipoledipole coupling can occur. In the case of non-adiabatic relaxation processes we rescale the velocity along the non-adiabatic coupling vector $\mathbf{d}_{k k^{\prime}}^{I}$ within the monomer where the state switch occurs. In the case of excitonic hops where a combined deexcitation in monomer $I$ and excitation in monomer $J$ occurs, we transfer the whole deexcitation energy of $\varphi_{i}^{I} \rightarrow \varphi_{i^{\prime}}^{I}$ to the monomer $J$, where it is used for the excitation $\varphi_{j}^{J} \rightarrow \varphi_{j^{\prime}}^{J}$. Residual deexcitation energy from $I$ is equally distributed on both participating monomers. If more energy is required to excite monomer $J$ than available from the deexcitation of monomer $I$, the required energy is withdrawn from the vibrational kinetic energy of both monomers. The state switch is rejected if the available energy is insufficient. The rescaling factors are obtained such that the total energy is conserved and that the change of kinetic energy is minimized in both monomers.

\section{Computational details}

An ideal double-stranded B-DNA (dsDNA) duplex containing 20 alternating Desoxyadenosines (dA) and Desoxythymidines (dT) per strand connected with the phosphate backbone $\left((\mathrm{dAdT})_{10}:(\mathrm{dAdT})_{10}\right)$ was generated and solvated in a $92 \AA$ cubic water box containing 25077 water and electrostatically neutralized by adding $19 \mathrm{Mg}^{2+}$ ions. The system was optimized and thermally equilibrated at $\mathrm{T}=300 \mathrm{~K}$ and $\mathrm{p}=1$ bar with periodic boundary conditions using the Gromacs 5 program package 66 67 with the Amber99 force-field parameters 68 . For the following dynamics simulations, carried out with our homemade software, we extracted a tube with a length and diameter of 80x50 ̊ containing the DNA, the $\mathrm{Mg}^{2+}$ ions and 4768 water molecules and froze the outer shell of water molecules in order to conserve volume and density.

In order to run $\mathrm{QM} / \mathrm{MM}$ simulations we selected 6 base pairs $\left((\mathrm{AT})_{3}:(\mathrm{AT})_{3}\right)$ lying in the center of the DNA strands as QM part and the rest (28 bases, sugar residue, phosphate backbone, water and ions) as MM part (cf. Fig. 1). The boundary bonds from the QM bases to the sugar residues were capped with hydrogens as link-atoms. The semi-empirical multi-reference configuration interaction OM2 method ${ }^{6970}$ as implemented in the MNDO99 software ${ }^{71 / 72}$ is used for the QM region and the Amber99 force-field for the MM region. Note that the formulation of McFISH method (cf. Sec. 2) is independent of the underlying quantum chemical as well as the molecular mechanical methods and can straight- forwardly extended to interface with external software providing the necessary quantities.

In the frame of the electrostatic embedding scheme the pointcharges of the MM system are included into the QM Hamiltonian as one-particle terms, while charges close to the boundary region are excluded to prevent over-polarization of the QM part. For the QM/MM McFISH simulations the QM system was further split into 3 sub-systems each containing two base pairs (3 (AT) : (AT)), where each QM sub-system is electrostatically coupled to the MM system as well as to the other two QM sub-systems.

The absorption spectrum and transition densities were obtained from the $\mathrm{QM} / \mathrm{MM}$ optimized geometry using the OM2/MR-CISD GUGA-CI method 73 , where for the $(\mathrm{AT})_{3}:(\mathrm{AT})_{3}$ system an active space of 48 occupied and 64 virtual orbitals was used and for each of the (AT) : (AT) sub-systems an active space including 18 occupied and 24 virtual orbitals has been chosen.

In order to obtain reasonable initial conditions, we have run a 100 ps long QM/MM trajectory in the electronic ground-state at constant temperature $(\mathrm{T}=300 \mathrm{~K})$. After thermal equilibration we sampled geometries and velocities in regular time intervals serving as starting points for our semi-classical QM/MM McFISH molecular dynamics. In total 75 trajectories have been propagated for $2 \mathrm{ps}$ starting at $t=-40 \mathrm{fs}$ in the electronic ground state with explicit inclusion of the irradiating laser field. For each sub-system we included the ground and 12 excited states. Note that the expansion in Eq. (15) in the manifold of 13 monomeric states would require the propagation of the electronic Schrödinger equation in the manifold of 2197 excitonic states. However, since $\mathbf{H}_{M C}$ contains only one- $\left(\mathbf{H}_{I}\right)$ and two-monomer $\left(\mathbf{V}_{I J}\right)$ terms and due to the orthogonality of the monomeric eigenstates $\varphi_{i}^{I}$, all matrix elements with three or more different indices in the set $a b \ldots z$ are zero. Therefore we removed matrix elements with three or more different indices by an uniform transformation which reduces the manifold to 469 states.

The Newtonian equations of motion for the nuclei are integrated using the velocity Verlet algorithm ${ }^{74}$ with a time step $\Delta t=0.1 \mathrm{fs}$ and the electronic Schrödinger equation was numerically integrated using the 4th order Runge-Kutta method with a step size of $10^{-5} \mathrm{fs}$. We have used a uniformly polarized Gaussianshaped laser pulse (Eq. (20) centered at $t_{0}=0$ with amplitude of $\varepsilon_{0}=0.08 \mathrm{GV} / \mathrm{m}$ corresponding to an intensity of $0.85 \mathrm{GW} / \mathrm{cm}^{2}$ and a full width at half maximum (FWHM) of $50 \mathrm{fs}$ ( $\sigma \approx 32 \mathrm{fs}$ ), giving rise to a broad spectral width. The central frequency $\omega_{0}$ of the laser pulse has been chosen as $4.7 \mathrm{eV}$ in order to access the lower energy band of the UV spectrum (see results below). While the pulse is active $\left(|\mathbf{E}(t)|>0.01 \varepsilon_{0}\right)$ no velocity rescaling upon electronic state switch is imposed, since we assume that all energy of the laser pulse is absorbed by the molecule. After the pulse has ceased energy rescaling is imposed as described in the previous section.

Analysis of Transition density. In order to classify the character of observed excitations we analyze the transition density. In the Supplementary Information (SI) a concise explanation of the obtained quantities used to characterize the excitations is provided. Briefly, by partitioning the transition density matrix into fragments (e.g. nucleobases or DNA strands) we obtain 
charge transfer (CT) values showing the percentage amount of CT character to an excited state ${ }^{75} \cdot 77$, the delocalization length (DL) indicating the number of fragments participating in an excitation ${ }^{59178}$, and an average position (POS value) ${ }^{79}$ and fractional transition density (FTD) ${ }^{7780}$ both providing the location of an excitation in terms of individual fragments. The CT value ranges from 0 , for no CT character, to 1 for a pure CT state. Throughout this work we use a cutoff value of CT $\geq 0.3$ for an excited state to be considered of having significant CT character. The DL value is 1 if the excitation is localized on one fragment only, and larger if the excitation is delocalized over multiple fragments. Usually this number is fractional, but throughout this work we will skip the decimal place and round the DL value to its closest integer for simplicity. Combining DL and CT values allows us to classify excited states according to (cf. Fig. S1] in SI):

1. $\mathrm{DL}=1$ and $\mathrm{CT}<0.3$ : Locally excited (LE) states, where the transitions are solely localized on one fragment,

2. DL $\geq 2$ and $\mathrm{CT}<0.3$ : Frenkel excitonic excited (EE) states, where multiple local transitions in two or more fragments contribute to an excitation,

3. $\mathrm{DL}=1$ and $\mathrm{CT} \geq 0.3$ : Charge transfer (CT) states, where a net charge is transferred between the fragments, and

4. $\mathrm{DL} \geq 2$ and $\mathrm{CT} \geq 0.3$ : Charge resonance (CR) states, where two electrons are resonantly exchanged between the fragments.

The possible POS value for two fragments range from 1 to 2 . If an excitation is solely localized at the first fragment the POS value becomes 1 , while it is 2 if the excitation is localized at the second. In both cases the DL value is 1 (local excitation, LE). In all other cases (EE, CT or CR) the POS value is 1.5.

\section{Results and Discussion}

We explore the excitation energy transport and nonradiative relaxation in the DNA duplex with the sequence $(\mathrm{dAdT})_{10}$ : (dAdT) $)_{10}$ embedded in water (Fig. 11) by employing the previously described QM/MM McFISH method. For this purpose we selected 6 alternating adenine-thymine base pairs $\left((\mathrm{AT})_{3}:(\mathrm{AT})_{3}\right)$ located in the center of the dsDNA as QM system (Fig. 1 1 right, further denoted as full QM system). We further partitioned the QM system into three subsystems consisting of 2 alternating base pairs (AT) : (AT) (further denoted as partitioned QM system, shaded areas in Fig. 11).. Each QM subsystem is calculated independently using the semi-empirical multi-reference configuration interaction OM2 (OM2/MRCI) method ${ }^{69 / 70}$ providing an efficient approach to simulate extended systems. Benchmark calculations show that the OM2 approach gives reliable results for the excited states of many organic molecules ${ }^{72181}$. The OM2 approach was successfully employed in a series of studies on both the static excited state properties and the nonadiabatic dynamics of nucleobases in isolated form 828283 , in solution $\sqrt{6384} \sqrt[86]{6}$, or embedded in DNA strands ${ }^{87}$. In studies on stacked nucleobase dimers and trimers it was also that semiempirical AM1 and PM3 methods provide reasonable energies for charge transfer states 88 . However, note that the presented McFISH method is generally applicable and can be combined with any electronic structure method, such as TDDFT or ADC(2). The residual bases, phosphate backbone, solvent and counter ions form the MM region described by a classical force field.

We have chosen this partition scheme in order to partially include quantum mechanical effects, which would not be present when separating the system between each base pair (e.g. shortrange effects) or along the helical axis between the strands (e.g. intra-strand proton/charge transfer). In order to allow excitation energy transfer between such coupled systems the electronic excited states are coupled via their transition dipole moments (transition dipole approximation, TDA). The TDA tends to overestimate excitonic coupling of close-lying chromophores due to short-range contributions stemming from molecular orbital overlap ${ }^{59}$. However, since another side-effect of this partition scheme is that the subsystem distance increases, the overestimation of excitonic coupling is partially compensated. Note that this effect is essentially due to error cancellation, but does not catch the effects due to the overlap of molecular orbitals. Furthermore, we wish to remark that there are certain aspects of the photodynamics that cannot be fully captured within the chosen model. Specifically, this concerns CT processes crossing the boundaries of the subsystems, since the total wave function is constructed from single-subsystem excited state wave functions. This issue of the exciton model has been recently addressed in the literature $\frac{89}{}$, but the proposed approach is much too demanding for the system studied here.

\subsection{UV/Vis spectroscopy}

In order to justify the partitioning and the TDA, we have calculated UV/Vis spectra from the QM/MM optimized geometries, both for the full QM system containing all 6 base pairs as well as for the partitioned QM system with each subsystem being excitonically coupled.

The comparison is presented in Fig. $2 \mathrm{~A}$ and $2 \mathrm{~B}$, respectively, and a detailed analysis of the lowest 20 electronically excited states is given in Tab. S1 and Tab. S2 of the Supplementary Information (SI). The obtained spectra for the full QM system (Fig. 2A) and the partitioned QM system using the TDA (Fig. 2B) are of similar shape and nearly every state in the full QM system is also present in partitioned system, although close lying states frequently interchange. Both spectra show two bands in the lower energy region, which are usually experimentally resolved as a single band, with the first being considered the red tail of the spectrum. We have chosen a small width for the convolution to separate these two bands, because they have distinct properties. The first band located between $4.6 \mathrm{eV}$ and $4.8 \mathrm{eV}$ is dominated by localized (red) or slightly delocalized (blue) excitations with $\pi \pi^{*}$ character localized at adenine and the excitations are distributed along the whole dsDNA. The second band, found between between 4.8 to $5.2 \mathrm{eV}$, is dominated by delocalized excitations over two or more nucleobases, and transitions having partly CT character (green) are found in the higher energy region of this band. The contribution of excitations dominantly located 


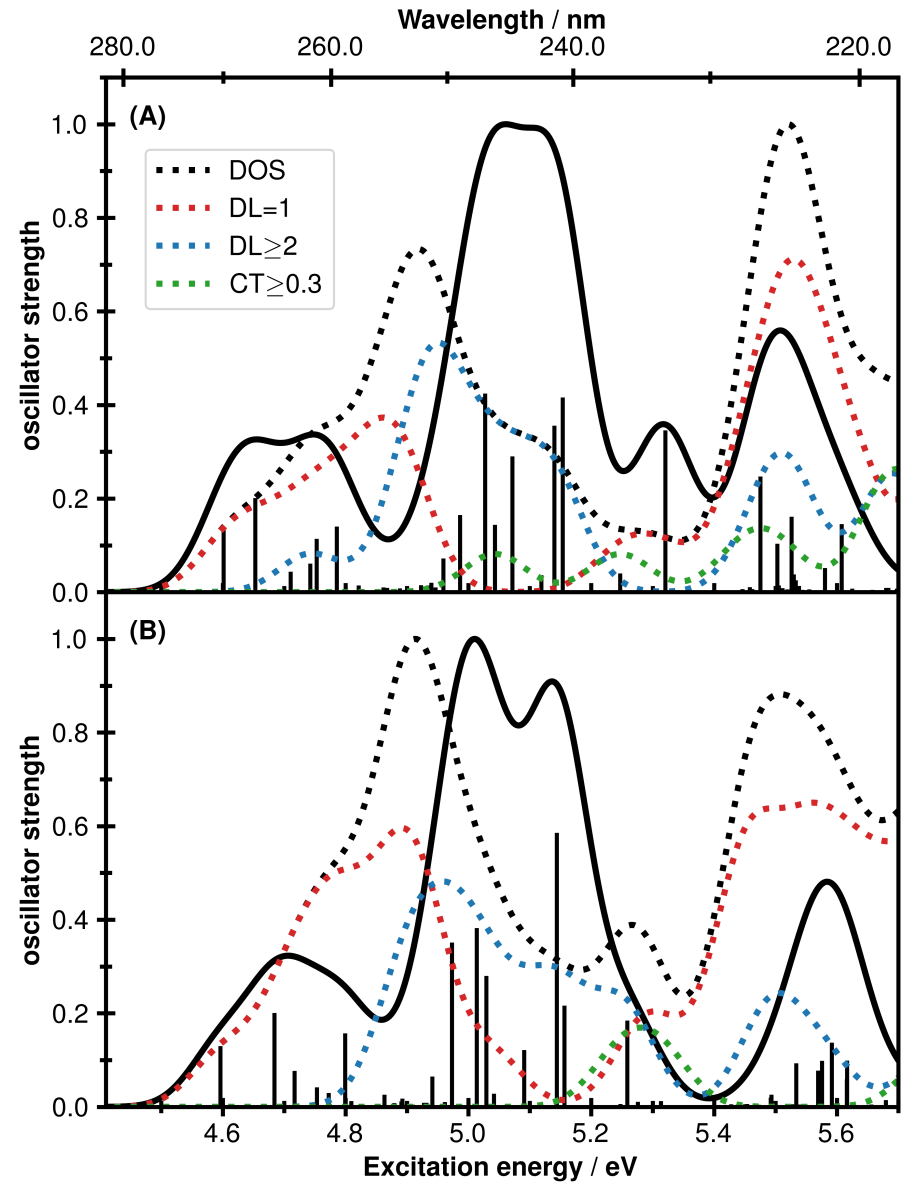

Fig. 2 UV/Vis spectra of the QM/MM optimized (dAdT) $)_{10}:(\mathrm{dAdT})_{10}$ doubled-strand DNA duplex embedded in water and ions. (A) QM system containing all 6 central $(\mathrm{AT})_{3}:(\mathrm{AT})_{3}$ base pairs. (B) QM region partitioned into 3 subsystems, each consisting of two base pairs, which are then excitonically coupled using the transition dipole approximation. The transitions (sticks) are convolved by a Lorentzian (black line, width 0.05 $\mathrm{eV}$ ). The dotted lines show the density of states (black) and its decomposition into localized states (blue), delocalized states (blue) and states with charge transfer contributions (green).

in thymine is low in the lower energy region and increases with higher energies (cf. Tabs. S1 and S2 in the SI).

The obtained spectra are in satisfactory agreement with experimental spectra of $(\mathrm{dAdT})_{10}:(\mathrm{dAdT})_{10}$ oligomers in buffered water solution (cf. Fig. S4 in the SI), which exhibit a broad band between 4.2-5.2 eV, where the lower end of this band has a small shoulder attributed to the $S_{0} \rightarrow S_{1}$ transition located at adenine ${ }^{61900}$. Our calculations are also in good agreement with the accurate ab-initio ADC(2)/MM calculations of the ATAT stacked tetramer embedded in a $(\mathrm{dAdT})_{6}:(\mathrm{dAdT})_{6}$ duplex and water 26 , where a broad band between 4.5 to $5.5 \mathrm{eV}$ with a maximum at 5.2 $\mathrm{eV}$ and an energetically lower-lying shoulder is found. In these calculations also DL and CT values have been analyzed, showing dominantly states of bright $\pi \pi^{*}$ character localized at one or two nucleobases, while the delocalization length is barely above 1.5. Dark $n \pi^{*}$ states are blue-shifted compared to spectra computed in-vacuo. When going to the maximum of this band the DL increases and states involving two or more monomers become more important. The most abundant exciton formed involves two nucleobases, leading to the conclusion that excited states of such stacked DNA bases are mostly localized or delocalized over not more than two stacked bases. Note that in our calculations we do not only have stacked bases but also base pairing. Therefore, we also observe excitonic states delocalized over three up to 4 bases, while the vertical intra-strand delocalization is dominantly limited to 2 bases.

Since the DNA is a flexible molecule and its secondary structure is highly dependent on the environment, the presented spectra represent only one snapshot of the possible spectral compositions. Therefore, we present in Fig. S4 of the SI the averaged spectrum of geometries extracted from the ground-state QM/MM trajectory. There, the localized and delocalized states are overlapping much more within the first band and thus excitonic states are accessible by the used laser pulse centered at $4.7 \mathrm{eV}$. The obtained spectrum is also in good agreement with the experimentally obtained spectrum of Bouvier et al.61 (gray dotted line in Fig. S4).

In summary, the good spectral agreement of full QM system and excitonically coupled partitioned QM system together with the good comparison to experimental and high-level ab-initio results makes us confident that our methodology is sufficiently accurate to simulate the nonradiative relaxation including the excitation energy transfer in dsDNA using our McFISH method, while being computationally efficient as well.

\subsection{Excited state energy transfer and relaxation}

In order to study the nonradiative relaxation of the excited states in dsDNA we perform McFISH simulations including the explicit interaction with the exciting laser field.

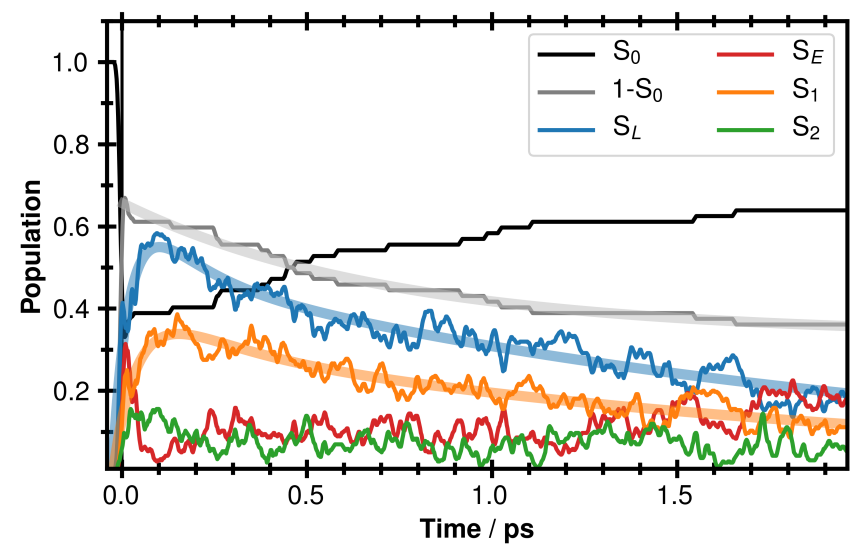

Fig. 3 Combined electronic state populations obtained from McFISH molecular dynamics simulations. The black and gray curves show the ground-state and total excited state population, respectively. The red and blue curves indicate the the sum of the population of locally $\left(S_{L}\right)$ and excitonic excited $\left(S_{E}\right)$ states. The green and orange curve are the sum of local (in respect to each subsystem) excited $S_{1}$ and $S_{2}$ states. The shaded thick curves indicate mono- and biexponential fits to the thin underlying curves.

As can be seen from Fig. 3 the laser pulse excites up to $67 \%$ of the trajectories (gray curve). Approximately $45 \%$ of this popu- 
lation is transferred to "excitonic" states ( $S_{E}$, blue curve) and the remaining trajectories are excited to "local" states ( $S_{L}$, red curve). Note that the termini "local" and "excitonic" have to be understood here on the basis of excited states of the subsystems, such that "local" excited states are limited to one nucleobase quartet and "excitonic" states are combinations of excited states in different subsystems. Therefore, no assignment of the excitation to individual nucleobases can be made. During the first $100 \mathrm{fs}$ most of the population of the $S_{E}$ states is transiently transferred to $S_{L}$ states, where the majority (38\%) reaches the energetically lowest subsystem state ( $S_{1}$, orange curve) within 200 fs. In the course of the dynamics the $S_{L}$ states decay slowly to the ground state approaching $18 \%$ after 2 ps. In total half of the initially excited trajectories returned to the ground-state within 2 ps.

In order to determine the lifetimes, we fitted biexponentially decaying model functions to the populations (shaded lines in Fig. 3 ), and all obtained parameters as well as the analytic form of the model functions are given in the SI. For the overall excited state lifetime (gray) we obtained a decay with time constants of $\tau_{2}=700 \mathrm{fs}$ and $\tau_{3} \approx 75 \mathrm{ps}$, giving rise to an average lifetime of $\langle\tau\rangle \approx 35$ ps. Note that, since the determined lifetime is far beyond the duration of our simulations, it can only serve as a rough estimate to the overall lifetime. For the $S_{L}$ states (red) time constants of $\tau_{1}=48$ fs for the population increase of these states, and $\tau_{2}=130 \mathrm{fs}$ and $\tau_{3}=2.06 \mathrm{ps}(\langle\tau\rangle=890 \mathrm{fs})$ for the decaying component were determined. The obtained values are in the same order of magnitude as obtained experimentally on (dAdT)duplexes $11 \mid 12$. For the heteropolymorphic $(\mathrm{dAdT})_{18}:(\mathrm{dAdT})_{18}$ duplexes Kohler et al. determined a lifetime of 51 ps, which is 3 times shorter than for the homopolymeric duplexes ${ }^{12}$, and femtosecond fluorescence spectroscopy reveals a wavelength dependent increase of the of the excited state lifetime from 0.5 ps at $330 \mathrm{~nm}$ to $3.3 \mathrm{ps}$ at $420 \mathrm{~nm} 11$. Thus the overall excited state lifetime is found to be much longer than for the isolated nucleobases, which exhibit radiationless decay times of a few 100 fs to 1 ps 91 96. Interestingly, the fast decaying component of the excited state population with a time constant of $0.7 \mathrm{ps}$ is close to the lifetime of 0.46 ps found for equimolar mixtures of deoxyadenosine monophosphate (dAMP) and thymidine monophosphate (TMP), and the fast decaying component $(0.13 \mathrm{ps})$ of the $S_{L}$ population is the same as for pure dAMP solutions ${ }^{97}$. These findings suggest and we will later show, that (1) the mechanism leading to the radiationless decay to the ground state in the early stages after photoexcitation involves monomer-like deactivation channels, which are already known from the bare nucleobases, and (2) the formation of and trapping in long-living delocalized excitonic states is responsible for the elongated excited state lifetime as indicated by the second slow decaying component of the excited state population. Therefore, at the end of simulation the excited state dynamics is largely governed by electronic states delocalized over several nucleobases.

While the electronic state population already shows elongation of the excited state lifetime compared to the monomers, the extent of the delocalization is not clear because multiple chromophores are involved in each subsystem and can participate in the formation of excimers. A nucleobase centered picture is pre-

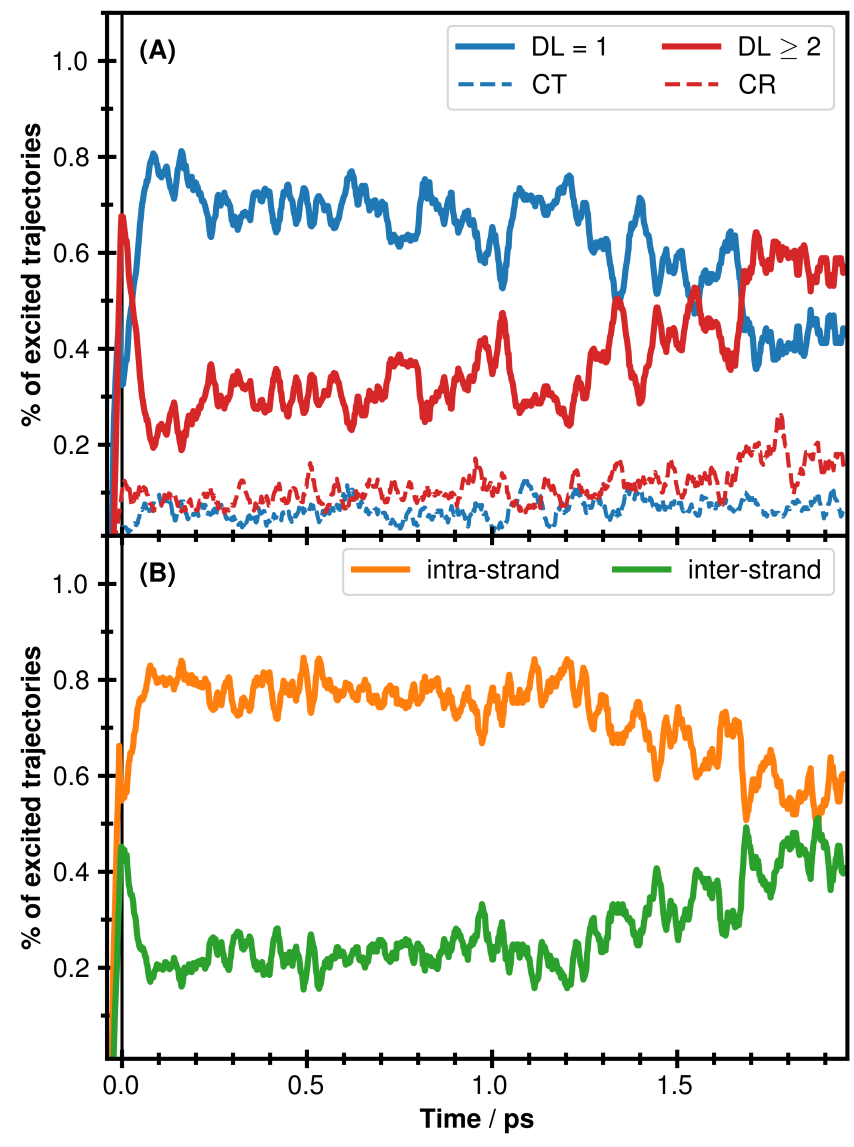

Fig. 4 Analysis of the transition density matrix (TDM) averaged with respect to trajectories being in the excited state. (A) Contribution of local excitations (blue) and delocalized excitations (red). The dotted curves denote charge separated states (CT $\geq 0.3$ ), being either charge transfer states (blue dotted curve, $\mathrm{DL}=1$ ) or charge resonance states (red dotted curve, $D L \geq 2$ ). (B) Location of the excitation obtained from POS values using each strand as a fragment. Inter-strand excitations possess 1.25 $<$ POS $<1.75$ and are delocalized between the strands and intra-strand excitation have POS $<1.25$ or POS $>1.75$, respectively, and are thus localized within one of the strands.

sented in Fig. 44 by calculating the delocalization length (DL) and charge transfer contributions (CT values) along each trajectory. Note that, in contrast to the electronic state populations shown in Fig. 3. all values shown Fig. 4 are now normalized to the number of trajectories that are in the excited state at a given time. This is reasonable since the transition density is only available for trajectories situated in the excited state and moreover emphasizes the processes taking place in the excited state.

As the used laser field has a central frequency of $4.7 \mathrm{eV}$, it lies in the center of the first band of the UV/Vis spectrum (Fig. 23) and due to its broad spectral width may populate a variety of excited states: localized states on adenine or thymine as well as ones delocalized over a few bases. Furthermore, delocalized excited states may be Frenkel excitons, charge resonance states or a mixture of them. While for the electronic state populations (Fig. 3 we found an equal distribution of local $\left(S_{L}\right)$ and excitonic $\left(S_{E}\right)$ excitations with respect to the subsystems, Fig. 4A shows that ac- 

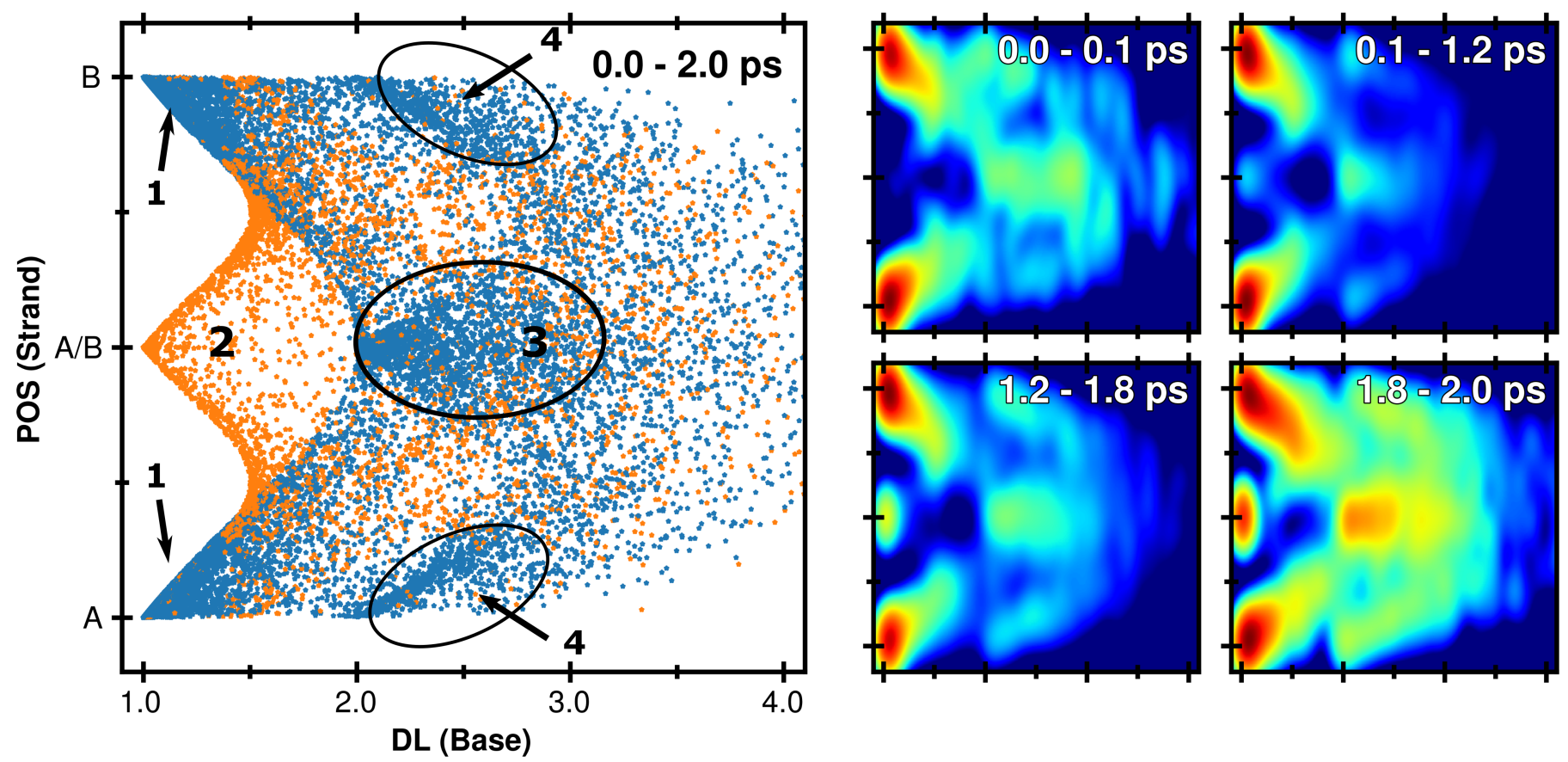

Fig. 5 Distribution of the excitation location (POS) with respect to the two DNA strands as a function of delocalization length (DL) with respect to the individual bases. Left panel: Distribution for the total simulation time. The orange dots denote charge transfer $(C T \geq 0.3$ and $D L=1)$ or charge resonance $(C T \geq 0.3$ and $D L \geq 2$ ) states, respectively. The inset numbers and marked regions are: (1) local excited (LE) states, (2) inter-strand charge transfer states, (3) inter-strand excitonic excited (EE) states and (4) intra-strand EE states. Right panels: Density of POS/DL points depicted for 4 time intervals showing the formation of delocalized inter- and intra-strand excitonic states. The density is shown on a logarithmic scale.

trajectories).

The diagram in Fig. 64 shows the average non-adiabatic coupling to the ground-state with respect to the delocalization length of the state the trajectories currently reside. For the short (orange) as well as the long (green) living trajectories the coupling to the ground-state vanishes exponentially with the increase of the degree of delocalization, such that excitations that are delocalized over more than 2 bases exhibit no relaxation to the ground-state. In the regime of localized excited states, however, the short trajectories show in average a three times higher non-adiabatic coupling to the ground-state. This indicates that the fast relaxation within the first 800 fs governed by the monomer-like photodynamics of localized excited states.

In order to be able to reach the monomer-like conical intersections with the ground-state the excitation needs to be localized sufficiently long on a single base and thus short trajectories should in average exhibit a longer retention time on a single base. Therefore we determined Fig. 63 the average length of time the fractional transition density (FTD) is constantly localized on a base ( $\max$ FTD $>0.8$ ). As can be seen, excitations in short trajectories reside in average three times longer (32 fs) localized on a single base than the long ones (11 fs). Additionally, as shown in Fig. S6 in the SI, switches of the location are less frequent in short trajectories. An even more pronounced trend is found for the time an excitation is in average situated within one strand (cf. Fig. S6 in the SI). While excitations in short trajectories are dominantly located in a single strand for $51 \mathrm{fs}$, excitations in long trajectories are in average only for $16 \mathrm{fs}$. This indicates in accor- dance with the previous findings (cf. Figs. 43 and 5) that in the early stages of the excited state dynamics the excitation energy is dominantly transported within the strand and that for longer living trajectories inter-strand excitation energy transport is becoming more important.

This finding is substantiated by the overall percentage time (total time in a given state divided by the total time in the excited state) each class of trajectories stays in a localized or delocalized state, respectively, as shown in Fig. 63. During their time in the excited state, short trajectories (left pie chart) are in average $92 \%$ in a localized state (blue) and $8 \%$ in a delocalized state, in contrast to long trajectories (right pie chart), which reside only for $74 \%$ in a localized and for $26 \%$ in a delocalized state. In average short trajectories stay for about 58 fs in a localized state, long trajectories only do so for 29 fs before switching to a delocalized state (not shown). The contribution of delocalized states is therefore two to three times larger in long living trajectories than in short ones, indicating the influence of the formation of excimers on the elongated excited state lifetime.

In summary, in trajectories which relax quickly to the electronic ground-state, the excitation is longer localized on a single nucleobase, thus providing a longer time to reach the well-known conical intersections of the monomeric nucleobases. This leads to short lifetimes comparable to those of the isolated monomers. In contrast, trajectories which stay for a long time in the excited state frequently switch the location of their excitation, and thus a previously excited monomer can return to its planar equilibrium geometry of the ground-state before reaching the conical 

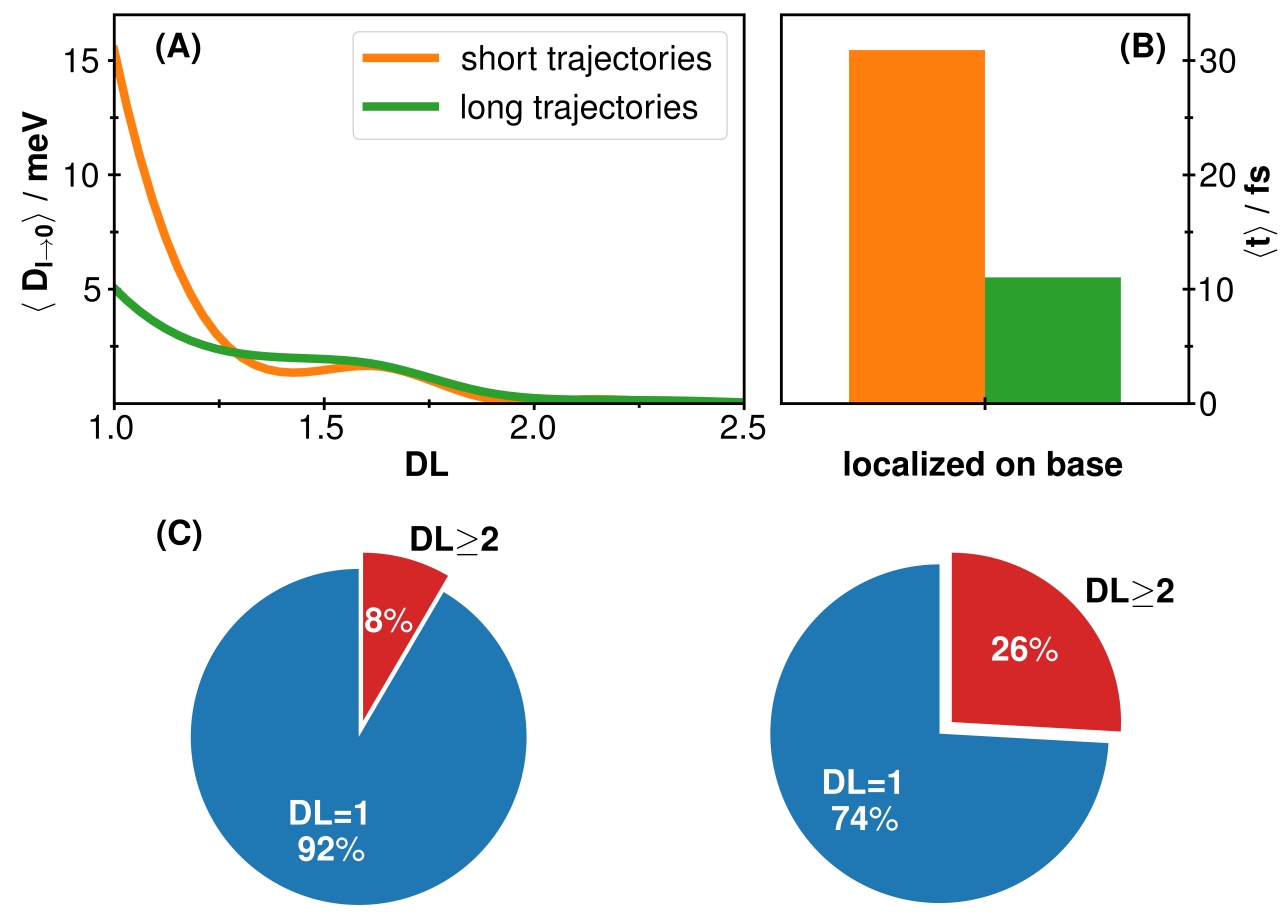

short trajectories

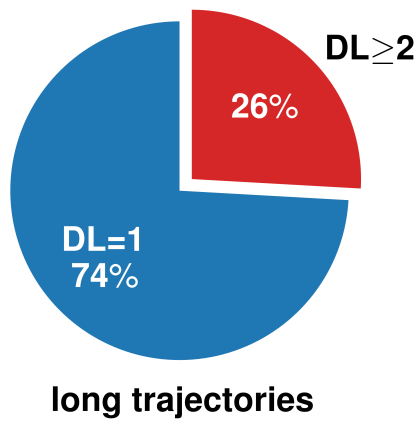

Fig. 6 (A) Average non-adiabatic coupling to the ground state as function of the delocalization length (DL). The plot indicates that short living trajectories (orange line) exhibit a strong coupling to the ground state that arises from states with localized excitations. The long living trajectories (green line) exhibit much lower coupling in the low DL range. The strength of the non-adiabatic coupling decreases in both cases with the delocalization length. (B) Average length of time trajectories reside in a state dominantly localized at a single base (max(FTD) $>0.8)$ showing that excitations in short (orange) trajectories are 3 times longer localized on a single base than long trajectories. (C) Total percentage of time short (left) and long (right) trajectories reside in localized (blue) and delocalized (red) states.

intersection. Hence, the average lifetimes of these trajectories are increased.

\subsection{Deactivation mechanisms}

In general the deactivation mechanism observed in the dynamics is basically comparable to the ones already known from the literature for adenine and thymine monomers 498,101 . Namely, we observe the out-of-plane movement of the methyl group of thymine associated with the barrierless internal conversion from a $\pi \pi^{*}$ to a $n \pi^{*}$ localized excited state ${ }^{98 / 100}$ and the out-of-plane puckering of adenine's amino group $101 / 102$. In Fig. 7 we present snapshots of an exemplary trajectory (a video showing the complete trajectory is provided in the SI). The laser pulse excites dominantly the central region of the QM region (40 fs). In the following 500 fs the excitation is propagated along both strands and all nucleobases until a localized $\pi \pi^{*}$ state is reached, which is converted to an $n \pi^{*}$ state in the following $200 \mathrm{fs}$. Finally, the trajectory relaxes to the electronic ground state at $780 \mathrm{fs}$ via a conical intersection involving the out-of-plane movement of the methyl group at thymine.

Radiationless internal conversion from bright localized $\pi \pi^{*}$ state in adenine-thymine base pairs $103-107$ and stacked dimers 28 to a CT state followed by proton transfer leading to a conical intersection with the ground state were reported. We also see regularly inter-strand proton transfer reactions as a two step tautomerization mechanism involving the proton transfer from the amino group of adenine to the hydrogen-bonded thymine oxygen and from the thymine $\mathrm{NH}$ hydrogen to the corresponding adenine's nitrogen. Although this process is photoinduced, in our simulations, it does not lead to a deactivation to the ground-state. In our case the tautomeric form is not created while the excitation is localized on the given base, but after the excitation has already been transferred to one of the neighboring bases, leaving a residual vibrational kinetic energy on the initially excited base. This energy is large enough to overcome the small barrier separating the canonical form from the the tautomeric one, which has been determined to be only $9-11 \mathrm{kcal} / \mathrm{mol}^{106} 108$. This "mutation" due to the inter-strand proton transfer is therefore an outcome of the excess kinetic energy introduced by excitonic hops and thus consequently a thermal effect initially caused by the external laser field. Such a transformation is in contrast to mutations located at a single strand (e.g. thymine dimerization), which can not easily repaired by using the second strand as template ${ }^{1 / 109}$. However, in our case the tautomerized state is not very stable and thus the protons are regularly transferred back, resulting in the canonical form being dominant during the dynamics. 


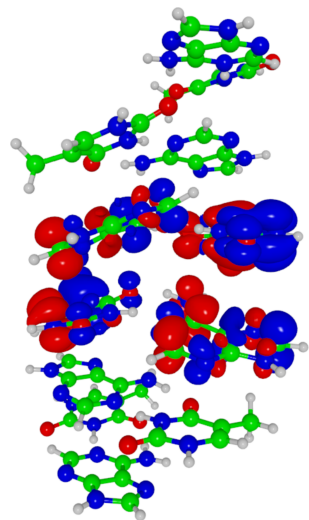

$40 \mathrm{fs}$

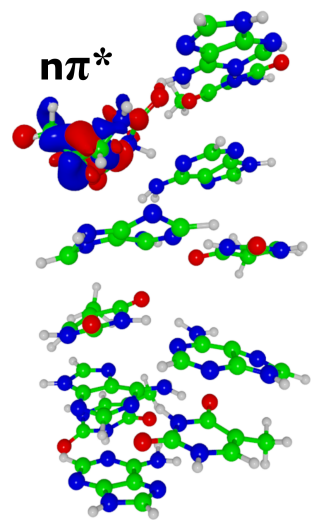

700 fs

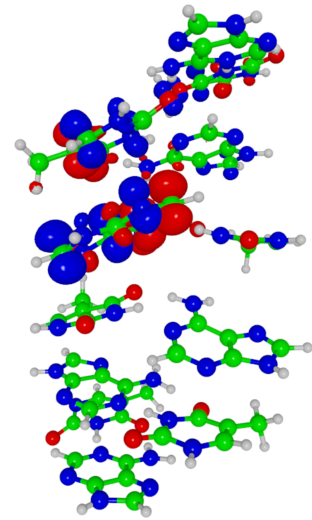

200 fs

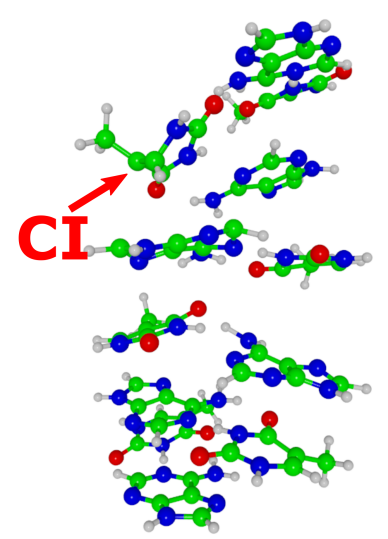

780 fs

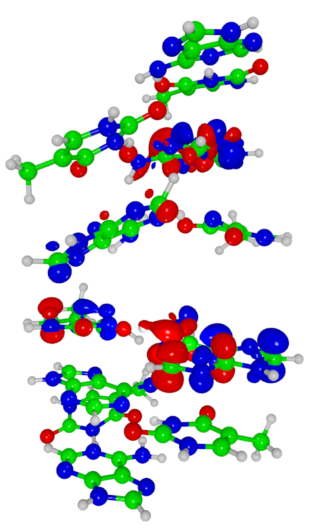

400 fs

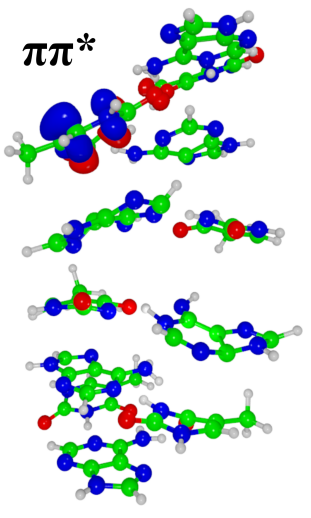

550 fs

Fig. 7 Snapshots and transition densities of the excited state where the trajectory currently resides, for an exemplary trajectory. The first frame (40 fs) shows the initial excitation of an excitonic state involving 4 nucleobases. The following two snapshots demonstrate the excitation energy transport along the DNA duplex, which is finally trapped in a local $\pi \pi^{*}$ excitation located at thymine (550 fs). The $\pi \pi^{*}$ state internally converts to an $n \pi^{*}$ state at $700 \mathrm{fs}$, followed by the deactivation to the ground-state via a methyl group out-of-plane puckering conical intersection at 780 fs.

\section{Conclusion}

With our newly developed McFISH method we introduce a versatile and generally applicable approach to simulate excitation energy transport in complex and extended molecular aggregates including all degrees of freedom by the combination of hybrid quantum/molecular mechanics (QM/MM) techniques and semi-classical surface-hopping dynamics on-the-fly. We have demonstrated our methodology by simulating the laser fieldinduced dynamics in the solvated double-stranded alternating $(\mathrm{dAdT})_{10}:(\mathrm{dAdT})_{10}$ DNA-oligomer. In our model the central 6 base pairs were treated by the semiempirical multi-reference (MR-CI) OM2 method and the remaining DNA as well as the solvating water and ions were described by a molecular mechanical force field. The QM region was further partitioned in 3 subsystems containing 2 base pairs each and afterwards electronically coupled through the transition dipole moments.

We have calculated the UV/Vis spectrum of the "full" QM model, considering all 6 base pairs as a single QM system including all quantum-mechanical effects, and compared it with the spectrum obtained for the partitioned system where the 3 sub-systems are coupled using the transition dipole approxima- tion. We have extensively analyzed and compared the transition density of the lowest 20 electronically excited states for the two models, finding a good agreement in the energetical position as well as the nature of the excited states. The obtained spectra are also in good agreement with available experimental data and high-level ab-initio calculations.

In accordance with previous experimental results ${ }^{12}$ we find after initial excitation of exciton states that the total excited-state population of the excited states is multi-exponentially decaying. Two time constants for the overall excited state lifetime have been obtained, the first being $700 \mathrm{fs}$, which resembles the lifetime of an equimolar mixture of dAMP (130 fs) and TMP 97 , and the second being $\sim 75 \mathrm{ps}$. From the obtained constants we estimate an average excited state lifetime of $\sim 36$ ps which is much longer than the monomeric deactivation lifetimes lying in the sub-ps regime.

A thorough analysis of the simulated trajectories allowed us to identify two groups exhibiting a distinctly different relaxation behavior:

(i) A substantial fraction of the trajectories undergoes an ultrafast internal conversion to the ground state in the early stages of the dynamics following the monomeric decay channels already 
known from investigations on the bare nucleobases ${ }^{94 \mid 110}$. These trajectories stay shorter in delocalized states and thus have more time to reach the conical intersections known for nucleobases.

(ii) The second group of trajectories decay much slower and is linked to the formation of excimers, where the excitation is delocalized either within one strand (intra-strand) or across both of them (inter-strand). We found that trajectories lasting for a long time ( $>1 \mathrm{ps}$ ) in the excited state exhibit also a dominant contribution of delocalized states involving two or more nucleobases. While in the early stages of the excitation the delocalization is dominantly distributed along one strand of the DNA duplex, it becomes increasingly delocalized over both strands and inter-strand charge resonance states become more important.

Our results provide a detailed molecular picture of the excited state relaxation and energy transfer processes in dsDNA. Overall, the simulation results are in good agreement with previously published experimental findings demonstrating that our QM/MM McFISH method is a reliable tool for the investigation of excitation energy transport in complex and large molecular multichromophoric aggregates. Therefore, we are planning to simulate and compare a variety of DNA sequences, such as homopolymeric chains, natural DNA as well as unusual DNA structures as found in e.g. i-motif DNA.

\section{Conflicts of interest}

There are no conflicts to declare.

\section{Acknowledgements}

We acknowledge the financial support within the ERC Consolidator Grant "DYNAMO" (Grant No. 646737).

\section{References}

1 G. P. Pfeifer, Y.-H. You and A. Besaratinia, Mutat. Res., 2005, 571, 19-31.

2 J. S. Taylor, Acc. Chem. Res., 1994, 27, 76-82.

3 A. Lamola and J. Eisinger, Proc. Natl. Acad. Sci. U.S.A., 1968, 59, 46.

4 R. Improta, F. Santoro and L. Blancafort, Chem. Rev., 2016, 116, 3540-3593.

5 C. T. Middleton, K. de La Harpe, C. Su, Y. K. Law, C. E. Crespo-Hernández and B. Kohler, Annu. Rev. Phys. Chem., 2009, 60, 217-239.

6 C. E. Crespo-Hernández, B. Cohen, P. M. Hare and B. Kohler, Chem. Rev., 2004, 104, 1977-2020.

7 J. Eisinger, M. Gueron, R. Shulman and T. Yamane, Proc. Natl. Acad. Sci. U.S.A, 1966, 55, 1015.

8 I. Vayá, T. Gustavsson, T. Douki, Y. Berlin and D. Markovitsi, J. Am. Chem. Soc., 2012, 134, 11366-11368.

9 I. Vayá, T. Gustavsson, F.-A. Miannay, T. Douki and D. Markovitsi, J. Am. Chem. Soc., 2010, 132, 11834-11835.

10 T. Takaya, C. Su, K. de La Harpe, C. E. Crespo-Hernández and B. Kohler, Proc.Natl. Acad. Sci., 2008, 105, 1028510290.

11 D. Onidas, T. Gustavsson, E. Lazzarotto and D. Markovitsi, Phys. Chem. Chem. Phys., 2007, 9, 5143-5148.
12 C. E. Crespo-Hernández, B. Cohen and B. Kohler, Nature, 2005, 436, 1141.

13 D. Markovitsi, Photochem. Photobiol., 2016, 92, 45-51.

14 A. Banyasz, T. Gustavsson, D. Onidas, P. Changenet-Barret, D. Markovitsi and R. Improta, Chem.-Eur. J., 2013, 19, 3762-3774.

15 D. Markovitsi, T. Gustavsson and I. Vayá, J. Phys. Chem. Lett., 2010, 1, 3271-3276.

16 W.-M. Kwok, C. Ma and D. L. Phillips, J. Phys. Chem. B, 2009, 113, 11527-11534.

17 N. K. Schwalb and F. Temps, Science, 2008, 322, 243-245.

18 E. R. Bittner, J. Photochem. Photobiol., A, 2007, 190, 328334.

19 R. Borrego-Varillas, G. Cerullo and D. Markovitsi, J. Phys. Chem. Lett., 2019, 10, 1639-1643.

20 I. Conti and M. Garavelli, J. Phys. Chem. Lett., 2018, 9, 2373-2379.

21 J. J. Nogueira, F. Plasser and L. González, Chem. Sci., 2017, 8, 5682-5691.

22 V. A. Spata, W. Lee and S. Matsika, J. Phys. Chem. Lett., 2016 , 7, 976-984.

23 L. Blancafort and A. A. Voityuk, J. Chem. Phys., 2014, 140, 03B602_1.

24 A. A. Voityuk, Photochem. Photobiol. Sci., 2013, 12, 1303 1309.

25 F. Plasser and H. Lischka, Photochem. Photobiol. Sci., 2013, 12, 1440-1452.

26 F. Plasser, A. J. Aquino, W. L. Hase and H. Lischka, J. Phys. Chem. A, 2012, 116, 11151-11160.

27 A. W. Lange and J. M. Herbert, J. Am. Chem. Soc., 2009, 131, 3913-3922.

28 F. Santoro, V. Barone and R. Improta, J. Am. Chem. Soc., 2009, 131, 15232-15245.

29 S. Tonzani and G. C. Schatz, J. Am. Chem. Soc., 2008, 130, 7607-7612.

30 I. Buchvarov, Q. Wang, M. Raytchev, A. Trifonov and T. Fiebig, Proc.Natl. Acad. Sci., 2007, 104, 4794-4797.

31 D. Markovitsi, T. Gustavsson and F. Talbot, Photochem. Photobiol. Sci., 2007, 6, 717-724.

32 F.-A. Miannay, Á. Bányász, T. Gustavsson and D. Markovitsi, J. Am. Chem. Soc., 2007, 129, 14574-14575.

33 R. Improta and V. Barone, Angew. Chem. Int. Ed., 2011, 50, 12016-12019.

34 R. Szabla, H. Kruse, P. Stadlbauer, J. Šponer and A. L. Sobolewski, Chem. Sci., 2018, 9, 3131-3140.

35 J. Garrec, C. Patel, U. Rothlisberger and E. Dumont, J. Am. Chem. Soc., 2012, 134, 2111-2119.

36 J. C. Tully, J. Chem. Phys., 1990, 93, 1061-1071.

37 A. Sisto, D. R. Glowacki and T. J. Martinez, Acc. Chem. Res., 2014, 47, 2857-2866.

38 A. Sisto, C. Stross, M. W. van der Kamp, M. O'Connor, S. McIntosh-Smith, G. T. Johnson, E. G. Hohenstein, F. R. Manby, D. R. Glowacki and T. J. Martinez, Phys. Chem. Chem. Phys., 2017, 19, 14924-14936. 
39 M. F. Menger, F. Plasser, B. Mennucci and L. González, J. Chem. Theory Comput., 2018, 14, 6139-6148.

40 R. Mitrić, J. Petersen, M. Wohlgemuth, U. Werner, V. Bonačić-Kouteckỳ, L. Wöste and J. Jortner, J. Phys. Chem. A, 2010, 115, 3755-3765.

41 R. Mitrić, J. Petersen and V. Bonačić-Kouteckỳ, Phys. Rev. A, 2009, 79, 053416.

42 R. van Grondelle and V. I. Novoderezhkin, Phys. Chem. Chem. Phys., 2006, 8, 793-807.

43 R. Hildner, D. Brinks, J. B. Nieder, R. J. Cogdell and N. F. van Hulst, Science, 2013, 340, 1448-1451.

44 G. S. Schlau-Cohen, Q. Wang, J. Southall, R. J. Cogdell and W. Moerner, Proc. Nat. Acad. Sci., 2013, 110, 10899-10903.

45 E. A. Jares-Erijman and T. M. Jovin, Nature Biotechnol., 2003, 21, 1387-1395.

46 E. Galperin, V. V. Verkhusha and A. Sorkin, Nature Methods, 2004, 1, 209-217.

47 K. A. Lukyanov, D. M. Chudakov, S. Lukyanov and V. V. Verkhusha, Nat. Rev. Mol. Cell Bio., 2005, 6, 885-890.

48 D. E. Dolmans, D. Fukumura and R. K. Jain, Nat. Rev. Cancer, 2003, 3, 380-387.

49 T. A. Theodossiou, A. R. Gonçalves, K. Yannakopoulou, E. Skarpen and K. Berg, Angew. Chem. Int. Ed., 2015, 54, 4885-4889.

50 J. F. Lovell, T. W. Liu, J. Chen and G. Zheng, Chem. Rev., 2010, 110, 2839-2857.

51 Y. Huang, F. Qiu, R. Chen, D. Yan and X. Zhu, J. Mater. Chem. B, 2020, 8, 3772-3788.

52 E. Brunk and U. Rothlisberger, Chem. Rev., 2015, 115, 62176263.

53 H. M. Senn and W. Thiel, Angew. Chem. Int. Ed., 2009, 48, 1198-1229.

54 A. Warshel and M. Levitt, J. Mol. Biol., 1976, 103, 227-249.

55 T. Förster, in Modern Quantum Chemistry, ed. O. Sinanoglu, Academic Press: New York, 1965, ch. Delocalized Excitation and Excitation Transfer.

56 M. Madjet, A. Abdurahman and T. Renger, J. Phys. Chem. B, 2006, 110, 17268-17281.

57 B. P. Krueger, G. D. Scholes and G. R. Fleming, J. Phys. Chem. $B, 1998,102,5378-5386$.

58 D. L. Andrews, Chem. Phys., 1989, 135, 195-201.

59 A. Czader and E. R. Bittner, J. Chem. Phys., 2008, 128, $01 \mathrm{~B} 613$.

60 B. Bouvier, T. Gustavsson, D. Markovitsi and P. Millié, Chem. Phys., 2002, 275, 75-92.

61 B. Bouvier, J.-P. Dognon, R. Lavery, D. Markovitsi, P. Millié, D. Onidas and K. Zakrzewska, J. Phys. Chem. B, 2003, 107, 13512-13522.

62 S. Hammes-Schiffer and J. C. Tully, J. Chem. Phys., 1994, 101, 4657-4667.

63 J. Petersen, M. Wohlgemuth, B. Sellner, V. Bonačić-Kouteckỳ, H. Lischka and R. Mitrić, Phys. Chem. Chem. Phys., 2012, 14, 4687-4694.

64 M. Wohlgemuth and R. Mitrić, J. Phys. Chem. A, 2016, 120,
8976-8982.

65 P. G. Lisinetskaya and R. Mitric, Phys. Rev. A, 2011, 83, 033408/1-033408/13.

66 M. J. Abraham, T. Murtola, R. Schulz, S. Páll, J. C. Smith, B. Hess and E. Lindahl, SoftwareX, 2015, 1, 19-25.

67 H. J. Berendsen, D. van der Spoel and R. van Drunen, Comput. Phys. Commun., 1995, 91, 43-56.

68 J. Wang, P. Cieplak and P. A. Kollman, J. Comput. Chem., 2000, 21, 1049-1074.

69 W. Weber and W. Thiel, Theor. Chem. Acc., 2000, 103, 495506.

70 W. Weber, PhD thesis, Universität Zürich, Switzerland, 1996.

71 W. Thiel, MNDO Program. Version 06/2010, 2010.

72 M. Korth and W. Thiel, J. Chem. Theory Comput., 2011, 7, 2929-2936.

73 A. Koslowski, M. E. Beck and W. Thiel, J. Comput. Chem., 2003, 24, 714-726.

74 W. C. Swope, H. C. Andersen, P. H. Berens and K. R. Wilson, J. Chem. Phys., 1982, 76, 637-649.

75 A. Luzanov and O. Zhikol, Int. J. Quantum Chem., 2010, 110, 902-924.

76 A. Luzanov and O. Prezhdo, Int. J. Quantum Chem., 2005, 102, 582-601.

77 S. Tretiak and S. Mukamel, Chem. Rev., 2002, 102, 31713212.

78 J. Pipek and P. G. Mezey, Int. J. Quantum Chem., 1988, 34, 1-13.

79 F. Plasser and H. Lischka, J. Chem. Theory Comput., 2012, 8, 2777-2789.

80 S. Fernandez-Alberti, D. V. Makhov, S. Tretiak and D. V. Shalashilin, Phys. Chem. Chem. Phys., 2016, 18, 10028-10040.

81 M. R. Silva-Junior and W. Thiel, J. Chem. Theory Comput., 2010, 6, 1546-1564.

82 Z. Lan, E. Fabiano and W. Thiel, J. Phys. Chem. B, 2009, 113, 3548-3555.

83 E. Fabiano and W. Thiel, J. Phys. Chem. A, 2008, 112, 68596863.

84 B. Heggen, Z. Lan and W. Thiel, Phys. Chem. Chem. Phys., 2012, 14, 8137-8146.

85 Z. Lan, Y. Lu, E. Fabiano and W. Thiel, ChemPhysChem, 2011, 12, 1989-1998.

86 Y. Lu, Z. Lan and W. Thiel, J. Comput. Chem., 2012, 33, 1225-1235.

87 Y. Lu, Z. Lan and W. Thiel, Angew. Chem. Int. Ed, 2011, 50, 6864-6867.

88 A. A. Voityuk, Chem. Phys. Lett., 2006, 427, 177-180.

89 X. Li, R. M. Parrish, F. Liu, S. I. Kokkila Schumacher and T. J. Martinez, J. Chem. Theory Comput., 2017, 13, 3493-3504.

90 E. Emanuele, D. Markovitsi, P. Millié and K. Zakrzewska, ChemPhysChem, 2005, 6, 1387-1392.

91 S. M. Parker, S. Roy and F. Furche, Phys. Chem. Chem. Phys., 2019, 21, 18999-19010.

92 M. Barbatti, A. J. Aquino, J. J. Szymczak, D. Nachtigallová, 
P. Hobza and H. Lischka, Proc. Natl. Acad. Sci., 2010, 107, 21453-21458.

93 J. J. Szymczak, M. Barbatti, J. T. Soo Hoo, J. A. Adkins, T. L. Windus, D. Nachtigallová and H. Lischka, J. Phys. Chem. A, 2009, 113, 12686-12693.

94 S. Ullrich, T. Schultz, M. Z. Zgierski and A. Stolow, J. Am. Chem. Soc., 2004, 126, 2262-2263.

95 S. Ullrich, T. Schultz, M. Z. Zgierski and A. Stolow, Phys. Chem. Chem. Phys., 2004, 6, 2796-2801.

96 H. Kang, K. T. Lee, B. Jung, Y. J. Ko and S. K. Kim, J. Am. Chem. Soc., 2002, 124, 12958-12959.

97 D. Markovitsi, A. Sharonov, D. Onidas and T. Gustavsson, ChemPhysChem, 2003, 4, 303-305.

98 G. Zechmann and M. Barbatti, J. Phys. Chem. A, 2008, 112, 8273-8279.

99 S. Perun, A. L. Sobolewski and W. Domcke, J. Phys. Chem. A, 2006, 110, 9031-9038.

100 S. Perun, A. L. Sobolewski and W. Domcke, J. Phys. Chem. A, 2006, 110, 13238-13244.

101 A. L. Sobolewski and W. Domcke, Eur. Phys. J. D, 2002, 20,
369-374.

102 S. Perun, A. Sobolewski and W. Domcke, Chem. Phys., 2005, 313, 107-112.

103 M. Dargiewicz, M. Biczysko, R. Improta and V. Barone, Phys. Chem. Chem. Phys., 2012, 14, 8981-8989.

104 S. Perun, A. L. Sobolewski and W. Domcke, J. Phys. Chem. A, 2006, 110, 9031-9038.

105 F. L. Gervasio, M. Boero and M. Parrinello, Angew. Chem. Int. Ed., 2006, 45, 5606-5609.

106 N. Shimizu, S. Kawano and M. Tachikawa, J. Mol. Struct., 2005, 735, 243-248.

107 L. Gorb, Y. Podolyan, P. Dziekonski, W. A. Sokalski and J. Leszczynski, J. Am. Chem. Soc., 2004, 126, 10119-10129.

108 H.-H. Ritze, P. Hobza and D. Nachtigallová, Phys. Chem. Chem. Phys., 2007, 9, 1672-1675.

109 I. Conti, L. Martínez-Fernández, L. Esposito, S. Hofinger, A. Nenov, M. Garavelli and R. Improta, Chem.-Eur. J., 2017, 23, 15177-15188.

110 E. Samoylova, H. Lippert, S. Ullrich, I. Hertel, W. Radloff and T. Schultz, J. Am. Chem. Soc., 2005, 127, 1782-1786. 